\title{
BOUNDARY STABILIZATION OF A HYBRID SYSTEM
}

\author{
Kangsheng Liu \\ Department of Applied Mathematics \\ Zhejiang University, Hangzhou, 310027, China. \\ ksliu@public.hz.zj.cn \\ Zhuangyi Liu \\ Department of Mathematics \& Statistics \\ University of Minnesota, Duluth, MN 55812, USA.
}

zliu@d.umn.edu

\begin{abstract}
In this paper, we consider the boundary stabilization of a degenerate hybrid system composed of an Euler-Bernoulli beam with a tip mass. It is proved that the system is exponentially stabilizable when the usual velocity feedback controls are applied at the end with the tip mass. We also establish time reversibility and spectral completeness of the closed-loop system.
\end{abstract}

Key Words: boundary stabilization, beam, hybrid system, backward wellposedness, spectral completeness, multiplier technique

AMS subject classification: 93D15, 35B37, 35B40

\section{INTRODUCTION}

We consider a hybrid system which consists of an Euler-Bernoulli beam linked to a rigid body. The system is governed by the following equations:

$$
\left\{\begin{array}{l}
\rho w_{t t}+p w^{\prime \prime \prime \prime}=0, \quad(x, t) \in(0, L) \times(0, \infty) \\
w(0, t)=w^{\prime}(0, t)=0 \\
J w_{t t}^{\prime}(L, t)+p w^{\prime \prime}(L, t)=g(t) \\
M w_{t t}(L, t)-p w^{\prime \prime \prime}(L, t)=h(t)
\end{array}\right.
$$

where prime represents the derivative with respect to the spacial variable $x$, $p, \rho>0$ are the elasticity modulus and mass density respectvely; $J$ is the rotatory inertia of the tip mass $\mathrm{M} ; g(t), h(t)$ are controls applied at the end $x=L$. 
There are many papers in the literature on the stabilizability of the system (1.1) including [3], and [7]-[8]. It was shown in [3] that using the feedback law

$$
\left\{\begin{array}{l}
g(t)=-\alpha w_{t}^{\prime}(L, t) \\
h(t)=-\beta w_{t}(L, t)
\end{array}\right.
$$

where $\alpha, \beta>0,(1.1)$ is strongly stabilizable, but not exponentially stabilizable. Moreover, Rao[7] proved the lack of exponential stabilizability for a general feedback law

$$
\left\{\begin{array}{l}
g(t)=-\alpha_{1} w(L, t)-\alpha_{2} w^{\prime}(L, t)-\alpha_{3} w_{t}(L, t)-\alpha_{4} w_{t}^{\prime}(L, t) \\
h(t)=-\beta_{1} w(L, t)-\beta_{2} w^{\prime}(L, t)-\beta_{3} w_{t}(L, t)-\beta_{4} w_{t}^{\prime}(L, t)
\end{array}\right.
$$

with $\alpha_{i}, \beta_{i}, i=1, \cdots, 4$ being any real numbers. In the same paper, He also obtained the exponential stabilizability of (1.1) for the high-order feedback law

$$
\left\{\begin{array}{l}
g(t)=w_{t}^{\prime \prime \prime}(L, t) \\
h(t)=-w_{t}^{\prime \prime}(L, t)
\end{array}\right.
$$

Recently, Rao in [8] studied the plate version of the hybrid system (1.1). When reduced to the beam problem, his result implies that when $J=0$ and $M, \alpha, \beta>$ $0,(1.1)$ is exponentially stabilizable by the feedback law (1.2). However, the case of $J>0$ and $M=0$ was left as an open question. The main purpose of this paper is to give an affirmative answer to this question. Moreover, we will show that the $C_{0}$ semigroup associated with the closed-loop system is actually a $C_{0}$ group and its infinitesimal generator has a complete system of generalized eigenfunctions.

\section{EXPONENTIAL STABILIZATION}

With $M=0$, the controlled system (1.1) with feedback law (1.2) takes the form

$$
\left\{\begin{array}{l}
\rho w_{t t}+p w^{\prime \prime \prime \prime}=0, \quad(x, t) \in(0, L) \times(0, \infty) \\
w(0, t)=w^{\prime}(0, t)=0 \\
J w_{t t}^{\prime}(L, t)+p w^{\prime \prime}(L, t)=-\alpha w_{t}^{\prime}(L, t) \\
p w^{\prime \prime \prime}(L, t)=\beta w_{t}(L, t)
\end{array}\right.
$$

The physical interpretation of this system is that the tip mass is small enough to be neglected, but not the rotatory inertia. This situation occurs when the beam is linked with a large, but very light antena. Let

$$
W=\left\{w \in H^{2}(0, L) \mid w(0)=w^{\prime}(0)=0\right\}, \quad V=L_{\rho}^{2}(0, L) .
$$

Define the Hilbert space

$$
\mathcal{H}=W \times V \times \mathbb{C}
$$

equipped with the inner product

$$
\left\langle\left(w_{1}, v_{1}, z_{1}\right),\left(w_{2}, v_{2}, z_{2}\right)\right\rangle_{\mathcal{H}}=\int_{0}^{L}\left(p w_{1}^{\prime \prime} \bar{w}_{2}^{\prime \prime}+\rho v_{1} \bar{v}_{2}\right) d x+J z_{1} \bar{z}_{2}
$$


Furthermore, we define an operator $\mathcal{A}$ in $\mathcal{H}$ by

$$
\begin{gathered}
\mathcal{D}(\mathcal{A})=\left\{\begin{array}{l|l}
(w, v, z) & \begin{array}{c}
w, v \in W, w \in H^{4}(0, L), \\
p w^{\prime \prime \prime}(L)=\beta v(L), z=v^{\prime}(L)
\end{array}
\end{array}\right\}, \\
\mathcal{A}(w, v, z)=\left(v,-\frac{p}{\rho} w^{\prime \prime \prime \prime},-\frac{1}{J}\left(p w^{\prime \prime}(L)+\alpha z\right)\right) .
\end{gathered}
$$

Let $Y=(w, v, z)$. Then system (2.1) can be written as an abstract evolution equation in $\mathcal{H}$

$$
\frac{d Y}{d t}=\mathcal{A} Y
$$

Theorem $1 \mathcal{A}$ generates a $C_{0}$-semigroup, $e^{t \mathcal{A}}$, of contractions on $\mathcal{H}$, and $\mathcal{A}^{-1} \in \mathcal{L}(\mathcal{H})$ is a compact operator.

Proof. Since for $z=(w, v, z) \in \mathcal{D}(\mathcal{A})$ we have

$$
\begin{aligned}
\operatorname{Re}\langle\mathcal{A} z, z\rangle_{\mathcal{H}} & =\operatorname{Re} \int_{0}^{L}\left[p v^{\prime \prime} \bar{w}^{\prime \prime}-p w^{\prime \prime \prime \prime} \bar{v}\right] d x-\left(p w^{\prime \prime}(L)+\alpha z\right) \bar{z} \\
& =-\beta|v(L)|^{2}-\alpha|z|^{2} \leq 0,
\end{aligned}
$$

Hence, $\mathcal{A}$ is dissipative. It is easy to verify that for any $\left(f_{1}, f_{2}, f_{3}\right) \in \mathcal{H}$, equation

$$
\mathcal{A}(w, v, z)=\left(f_{1}, f_{2}, f_{3}\right), \quad(w, v, z) \in \mathcal{D}(\mathcal{A})
$$

has unique solution such that

$$
\|(w, v, z)\|_{\mathcal{H}} \leq M\left\|\left(f_{1}, f_{2}, f_{3}\right)\right\|_{\mathcal{H}},
$$

where the constant $M>0$ is independent of $\left(f_{1}, f_{2}, f_{3}\right)$. Therefore, $\mathcal{A}^{-1} \in$ $\mathcal{L}(\mathcal{H}), 0 \in \rho(\mathcal{A})$, and $\mathcal{A}$ is closed. It follows that the range $R(\lambda-\mathcal{A})=$ $\mathcal{H}$ for sufficiently small $\lambda>0$. By Theorem 4.6 in $[5], \overline{\mathcal{D}(\mathcal{A})}=\mathcal{H}$. The generation of $C_{0}$-semigroup now follows from the Lumer-Phillips theorem. By the compactness of embedding $H^{2}(0,1) \hookrightarrow C^{1}[0,1]$, we know that $\mathcal{A}^{-1}$ is also a compact operator.

It is clear that exponential stabilizability holds if $e^{t \mathcal{A}}$ is exponentially stable. We will employ the following frequency domain theorem for exponential stability of a $C_{0}$-semigroup of contractions on a Hilbert space [[1],[2], [6]]:

Lemma 2.1 $A C_{0}$-semigroup $e^{t \mathcal{A}}$ of contractions on a Hilbert space is exponentially stable if and only if

$$
\rho(\mathcal{A}) \supset\{i \lambda \mid \lambda \in \mathbb{R}\} \equiv i \mathbb{R}
$$

and

$$
\overline{\lim }_{|\lambda| \rightarrow \infty}\left\|(i \lambda-\mathcal{A})^{-1}\right\|<\infty
$$


Now, we are ready to state the main result in this section.

Theorem 2 The semigroup $e^{t \mathcal{A}}$ defined above is exponentially stable.

Proof. We only need verify conditions (2.7) and (2.8).

(i) Suppose (2.7) is false. Then, there exsit a nonzero $\lambda \in \mathbb{R}$ and $Y \in \mathcal{D}(\mathcal{A})$ with $\|Y\|_{\mathcal{H}}=1$ such that

$$
(i \lambda-\mathcal{A}) Y=0 .
$$

Take real part of the inner product of (2.9) with $z$ in $\mathcal{H}$, then apply (2.6). We obtain that

$$
v(L)=z=0 .
$$

Thus, (2.9)-(2.10) can be reduced to the following initial value problem

$$
\left\{\begin{array}{l}
-\lambda^{2} \rho w+p w^{\prime \prime \prime \prime}=0 \\
w(L)=w^{\prime}(L)=w^{\prime \prime}(L)=w^{\prime \prime \prime}(L)=0 .
\end{array}\right.
$$

There is nothing but $w=0$. Furthermore, $v=z=0$ follows from the first and third equation in (2.9). This contradicts to $\|Y\|_{\mathcal{H}}=1$.

(ii) Suppose (2.8) is false. Then by the Resonance Theorem, there exist a sequence of real numbers $\lambda_{n} \rightarrow \infty$ and a sequence of vectors $Y_{n}=\left(w_{n}, v_{n}, z_{n}\right) \in$ $\mathcal{D}(\mathcal{A})$ with $\left\|Y_{n}\right\|_{\mathcal{H}}=1$ such that

$$
\left\|\left(i \lambda_{n} I-\mathcal{A}\right) Y_{n}\right\|_{\mathcal{H}} \rightarrow 0 \text { as } n \rightarrow \infty .
$$

i.e.,

$$
\begin{array}{rlrl}
i \lambda_{n} w_{n}-v_{n} & \equiv f_{n} \rightarrow 0 & \text { in } W, \\
i \lambda_{n} \rho v_{n}+\frac{p}{\rho} w_{n}^{\prime \prime \prime \prime} & \equiv g_{n} \rightarrow 0 & \text { in } V . \\
i \lambda_{n} z_{n}+\frac{1}{J}\left(p w^{\prime \prime}(L)+\alpha z_{n}\right) & \equiv h_{n} \rightarrow 0 & & \text { in } \mathbb{C} .
\end{array}
$$

In view of (2.6) and (2.12), we have

$$
\left|v_{n}(L)\right|, \quad\left|z_{n}\right| \rightarrow 0
$$

which further implies that

$$
\left|w_{n}^{\prime \prime \prime}(L)\right|,\left|\lambda_{n} w_{n}^{\prime}(L)\right|,\left|\lambda_{n} w_{n}(L)\right| \rightarrow 0 .
$$

In what follows we first show that

$$
\left|w^{\prime \prime}(L)\right| \rightarrow 0
$$

Solve $v_{n}$ from equation (2.13) and substituting it into (2.14), we have

$$
-\lambda_{n}^{2} w_{n}+\frac{p}{\rho} w_{n}^{\prime \prime \prime \prime}=g_{n}+i \lambda_{n} f_{n}
$$


Denote $\phi_{n}=\sqrt{\left|\lambda_{n}\right|}$ and $\gamma=(\rho / p)^{1 / 4}$. We take the inner product of $(2.19)$ with $\frac{1}{\phi_{n}} e^{-\gamma \phi_{n}(L-x)}$ in $L^{2}(0, L)$ to get

$$
\begin{aligned}
& \left\langle-\phi_{n}^{3} w_{n}, \rho e^{-\gamma \phi_{n}(L-x)}\right\rangle+\left\langle\frac{p}{\rho} w_{n}^{\prime \prime \prime \prime}, \frac{1}{\phi_{n}} e^{-\gamma \phi_{n}(L-x)}\right\rangle \\
= & \left\langle g_{n}+i \lambda_{n} f_{n}, \frac{1}{\phi_{n}} e^{-\gamma \phi_{n}(L-x)}\right\rangle .
\end{aligned}
$$

Clearly, the inner product on the right-hand side of (2.20) converges to zero. After integrating by parts four times to the second inner product on the lefthand side of (2.20), we can cancel the resulting inner product with the first inner product on the left-hand side of (2.20). Using the boundary conditions of $w_{n}$ at $x=0$ in (2.1) and at $x=L$ in (2.17), we rewrite $(2.20)$ as

$$
\frac{p}{\rho} \phi_{n} e^{-\gamma L \phi_{n}}\left(-\frac{w_{n}^{\prime \prime \prime}(0)}{\phi_{n}^{2}}+\gamma \frac{w_{n}^{\prime \prime}(0)}{\phi_{n}}\right)-\frac{p \gamma}{\rho} w_{n}^{\prime \prime}(L) \rightarrow 0 .
$$

From equation (2.14), we see that $w_{n} / \lambda_{n}$ is bounded in $H^{4}(0, L)$. Applying the trace theorem, we obtain

$$
\frac{p}{\rho} \phi_{n} e^{-\gamma L \phi_{n}}\left|-\frac{w_{n}^{\prime \prime \prime}(0)}{\phi_{n}^{2}}+\gamma \frac{w_{n}^{\prime \prime}(0)}{\phi_{n}}\right| \leq C \phi_{n} e^{-\gamma L \phi_{n}} \rightarrow 0 .
$$

The claim in (2.18) follows from (2.21)-(2.22). With these boundary conditions of $w_{n}$ at hand, we now take the inner product of (2.19) with the standary multiplier $x w_{n}^{\prime}$ in $L^{2}(0, L)$. A straight forward calculation via integration by parts leads to

$$
\frac{\rho}{2}\left\|\lambda_{n} w_{n}\right\|^{2}+\frac{3 p}{2}\left\|w_{n}^{\prime \prime}\right\|^{2} \rightarrow 0
$$

This, together with equation (2.13), yields

$$
\left\|w_{n}^{\prime \prime}\right\|,\left\|v_{n}\right\| \rightarrow 0
$$

In summary, we get that $\left\|Y_{n}\right\|_{\mathcal{H}}$ converges to zero. A contradiction.

\section{BACKWARD WELLPOSEDNESS AND SPECTRAL COMPLETENESS}

In this section, by means of theory developed in Liu and Russell [4], we will prove the following:

Theorem $1 \mathcal{A}$ generates a $C_{0}$ group and has a complete system of generalized eigenfunctions.

Proof. From discussions in [4],§3,4, we need prove that there exist $T, \delta>0$ such that

$$
\int_{0}^{T}\left\|e^{t \mathcal{A}} Y_{0}\right\|_{\mathcal{H}}^{2} d t \geq \delta\left\|Y_{0}\right\|_{\mathcal{H}} \quad \forall Y_{0} \in \mathcal{D}(\mathcal{A}) .
$$


Set $(w(\cdot, t), v(\cdot, t), z(t))=e^{t \mathcal{A}} Y_{0}, E(t)=\frac{1}{2}\left\|e^{t \mathcal{A}} Y_{0}\right\|_{\mathcal{H}}^{2} . \quad$ For $Y_{0}=\left(\xi, \eta, z_{0}\right) \in$ $\mathcal{D}(\mathcal{A})$, we know that

$$
\left\{\begin{array}{l}
w \in C^{2}([0, \infty) ; V) \cap C^{1}([0, \infty) ; W) \cap C\left([0, \infty) ; H^{4}(0, L)\right) \\
w_{t}=v, z(t)=w_{t}^{\prime}(L, t)
\end{array}\right.
$$

and $w$ satisfies (2.1). Thus,

$$
E(t)=\frac{1}{2} \int_{0}^{L}\left(\left|p w^{\prime \prime}(x, t)\right|^{2}+\left|\rho w_{t}(x, t)\right|^{2}\right) d x+\frac{1}{2} J\left|w_{t}^{\prime}(L, t)\right|^{2} .
$$

We may assume without loss of generality that $w(x, t)$ is real-valued. Multiplying the first equation of $(2.1)$ by $w_{t}$ and integrating by parts, we obtain

$$
E(0)-E(t)=\int_{0}^{t}\left[\beta w_{t}(L, s)^{2}+\alpha w_{t}^{\prime}(L, s)^{2}\right] d s
$$

It follows that

$$
T E(0)-\int_{0}^{T} E(t) d t=\int_{0}^{T}(T-t)\left[\beta w_{t}(L, t)^{2}+\alpha w_{t}^{\prime}(L, t)^{2}\right] d t
$$

We now multiply the first equation of $(2.1)$ by $(T-t) x w^{\prime}$. Then we integrate by parts to get

$$
\begin{array}{r}
\int_{0}^{T}(T-t)\left[\rho w_{t}(L, t)^{2}+L p w^{\prime \prime}(L, t)^{2}\right] d t+2 T \int_{0}^{L} x \xi^{\prime} \eta d x \\
\leq 2 \int_{0}^{T}(T-t) w^{\prime}(L, t)\left[L \beta w_{t}(L, t)-p w^{\prime \prime}(L, t)\right] d t+C_{1} \int_{0}^{T} E(t) d t
\end{array}
$$

where $C_{1}$ is some positive number independent of $(\xi, \eta)$. Combination of (3.5) and (3.6) yields

$$
T E(0) \leq-2 T \int_{0}^{L} x \xi^{\prime} \eta d x+C_{T} \int_{0}^{T} E(t) d t
$$

for some $C_{T}>0$ independent of $(\xi, \eta)$. Applying a standard compactness argument, we can now obtain the desired estimate.

\section{References}

[1] Gearhart, L. M. (1978). Spectral theory for contraction semigroups on Hilbert space. Trans. Amer. Math. Soc., 236:385-394.

[2] Huang, F. (1985). Characteristic condition for exponential stability of linear dynamical systems in Hilbert spaces. Chinese Ann. of Diff. Eqs, 1:43-56.

[3] Littman, W. and Markus, L. (1988). Stabilization of a hybrid system of elasticity by feedback damping. Ann. Mat. Pura Appl., 52:281-330. 
[4] Liu, K. and Russell, D. L. (1998). Exact controllability, time reversibility and spectral completeness of linear systems in Hilbert spaces. preprint.

[5] Pazy, A. (1983). Semigroups of Linear Operators and Applications to Partial Differential Equations. Springer, New York.

[6] Prüss, J. (1984). On the spectrum of $C_{0}$-semigroups. Trans. Amer. Math. Soc., 284:847-857.

[7] Rao, B. (1995). Uniform stabilization of a hybrid system of elasticity. SIAM J. Cont. Optim., 33:440-454.

[8] Rao, B. (1998). Stabilization of a elastic plates with dynamical boundary control. SIAM J. Cont. Optim., 36:148-163. 